\title{
Integer-dimensional fractals of nonlinear dynamics
}

Fractal dimension was developed as a quantitative measure of complex, irregular
objects, essentially because it objects, essentially because it
had been accepted that fractals had been accepted that fractals
could not be characterised by could not be characterised by
integer dimensions. Dr Zonglu integer dimensions. Dr Zonglu
$\mathrm{He}$, a Professor of Economics at Kaetsu University, is challenging this basic concept with her novel finding that fractals actually can occur in integer dimension space-times. Her research demonstrates the consistency of both fractional and topological dimensions. Moreover, she discovers the control mechanisms of fracte beffects of nonlinear dynamics.

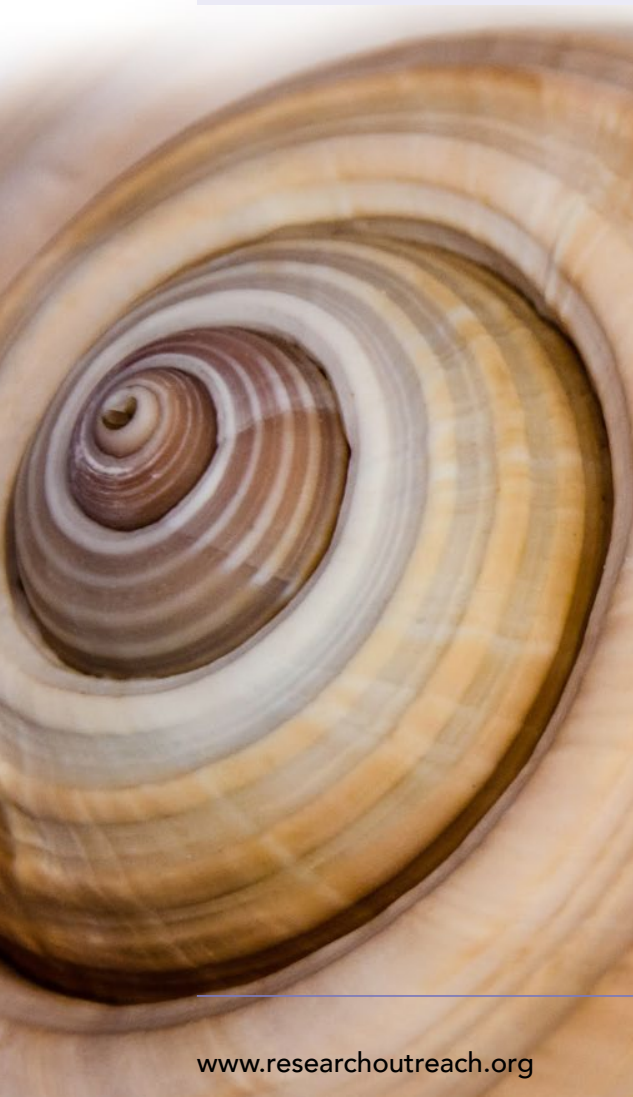

ractals are never ending patterns. - They can be curves or geometric figures, but each part appears a property known as self-similarity. They are created by the iterative repetition of a process or function. Fractals are images of dynamic systems diviven by recursion the image of chaos. Fractal patterns can snowflakes, seashells, flowers, trees, coastlines and alaxies Abstract fractals can be generated by computing an equation over and over. Fractals can used to model structures where patterns recur repeatedly at gradually smaller scales. They can also be used to describe random or chaotic phenomena such as crystal growth and fluid turbulence. Fractals also have applications in economics, such as modelling market price fluctuation or market risk.

FRACTAL DIMENSION ometric shapes have dimensions. For example, a point has a dimension , a line has a dimension of of 2 a volume has a
a dimension of 3 , we can derive we can derive shapes such

as cones and spheres. In the same way, fractals also have dimensions. These provide a measure of how complicated the fractal is. The fractal index, however, can have non-integer values e.g. a curve with a fractal dimension of 1.1 will perform like a one-dimensional line, whil curve with a fractal dimension of 1.9 (nits throngh space a

Fractal dimension was developed as a quantitative measure of complex, it was accepted that fractals could not be characterised by integer dimensions. Dr Zonglu He, a Professor of Economics at Kaetsu University, is challenging this basic concept with her novel finding that fractals actually can occur in integer dimensional space times.

\section{RESEARCH BACKGROUND} In the late 1990s, Professor He was initially interested in statistics, particularly stochastic (random) processes, and time series analysis. At that time, a major discovery was that economic cumulative effects of noise (such as money demand-supply shocks - sudden temporary increases or decreases in demand and supply) caused by the so-called 'unit root' structure. (A unit root is a randomly determined trend in a time series; its occurrence shows an unpredictable systematic pattern. This finding challenged traditional thinking of stationary fluctuations about a deterministic trend where money demand-supply shocks were assumed to have no long-term impact on the economy. A structural break (an unexpected change in the economy prompted by a sudden event such as a war or a change in government policy) as long memory, a feature of statistical

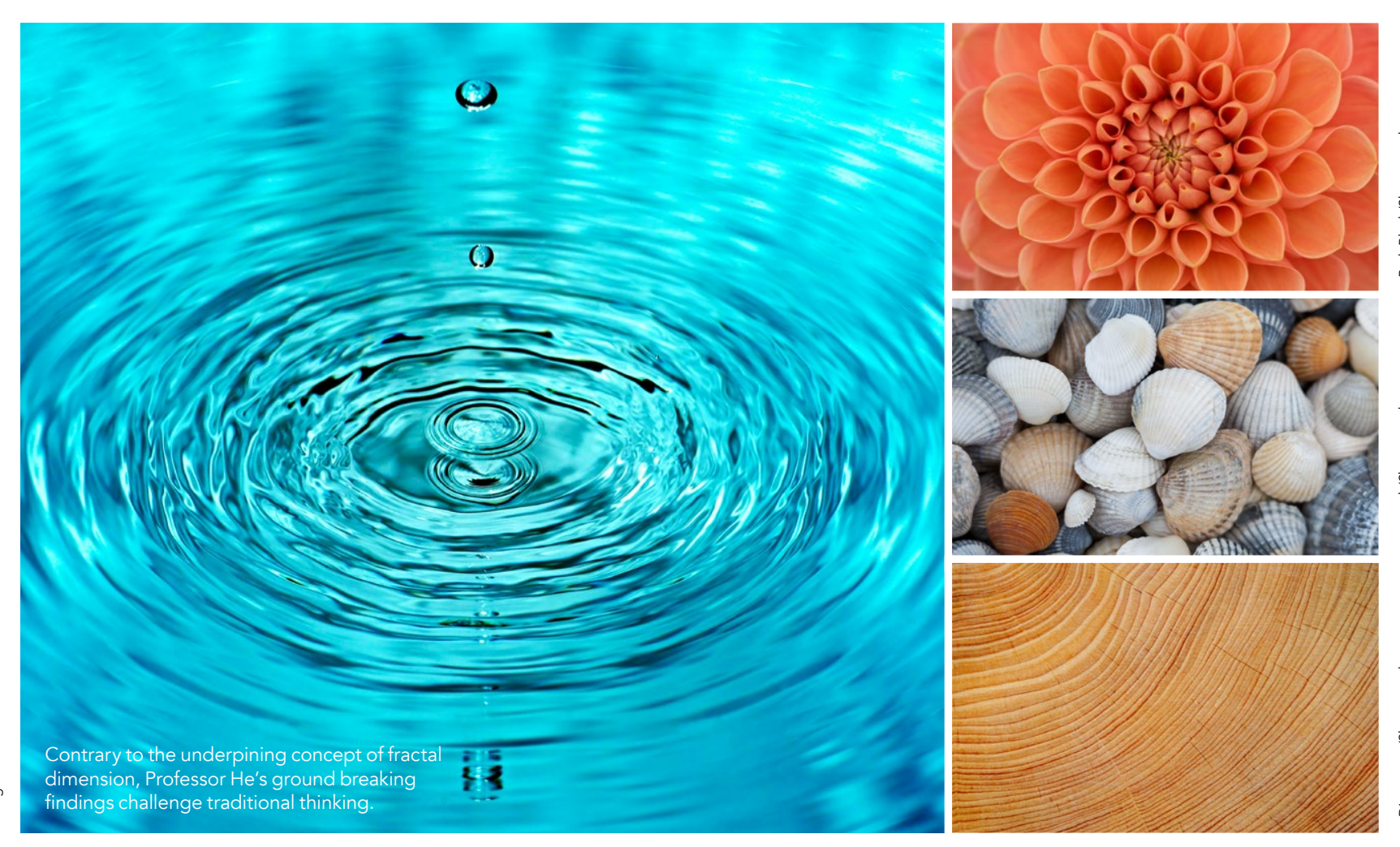

time series involving persistently strong uutocorrelation between remote as displaying unit root behaviour. It was difficult to say whether either one of these data features actually results in economic fluctuations. While a plethora of analytica literature exists regarding the testing of the data features, little attention has been paid to their causes.

THE NONLINEAR AUTOREGRESSIVE INTEGRATED (NLARI) MODEL Professor He pointed out that nonexplain what croas fracts bet and controls fractal levels. She believed that the solution to these issues would be found in modelling the processes involved in generating the data in different situations based on physica laws. If not, the model parameters would not have explicitly physical effects. She went on to highlight that an interdisciplinary unified time series model was required in order to systematically understand the essentia mechanisms of time series processes in the real world. This led to Professor $\mathrm{H}$ inventing the nonlinear autoregressive integrated (NLARI) model.

Professor He explains that she created the NLARI model to, "explore whether the data generating process for integer
Using the NLARI framework, Professor He was able to explain the nature and causes of unit roots and trend breaks.

dimensional space could exhibit fractal behaviour over time, particularly in the parameter ranges of nonlinear stochastic and deterministic dynamics". She derived the NLARI model by applying Newton's second law (force = mass $x$ acceleration) to stochastic selfrestoring systans in order to achieve of economic variables.

\section{RESEARCH FINDINGS} Using the NLARl framework, Professor He was able to explain the nature

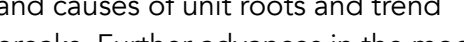
breaks. Further advances in the model meant that Professor He could clarify the NLARI nonlinear dynamics, by obtaining the analytic solutions of the determisictic system, and develop

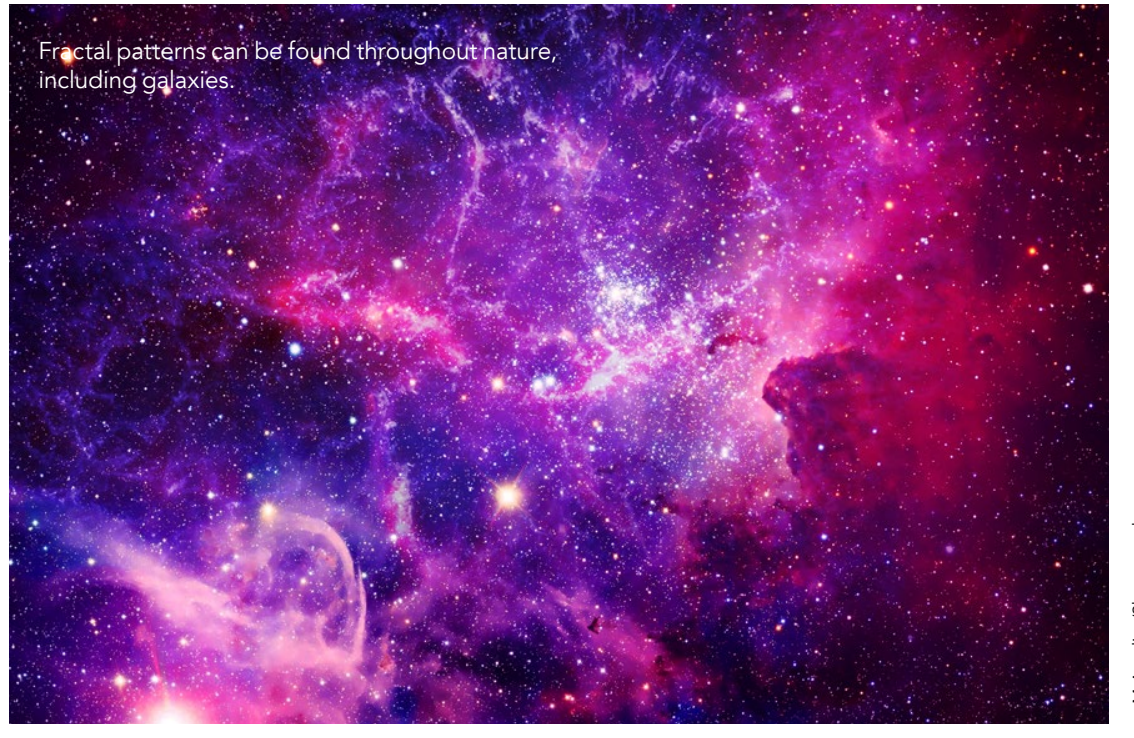


parameter estimation and hypothesis to investigate integer-dimens abo

fractal behaviours and their control

mechanisms as well as the relationships

between fractal and dynamic

behaviours, and the effects of stochastic

disturbances on fractals.

\section{CONSISTENCY WITH}

OPOLOGICAL DIMENSION

This study reveals that it is possible

for fractal dimension to be consistent

with topological dimension. Professor

He found that the properties of typica nonlinear dynamics, such as stable fixed point, periodic and aperiodic oscillations, and chaos, and those long-range dependence, together with elf-simility and power law were the same as the properties of the datagenerating process in integer dimensio pace-times. (A power law is a functional elationship between two quantities, where independent of the initial size of the quantities, one quantity varies as a power of another.

Professor He discovered that long-
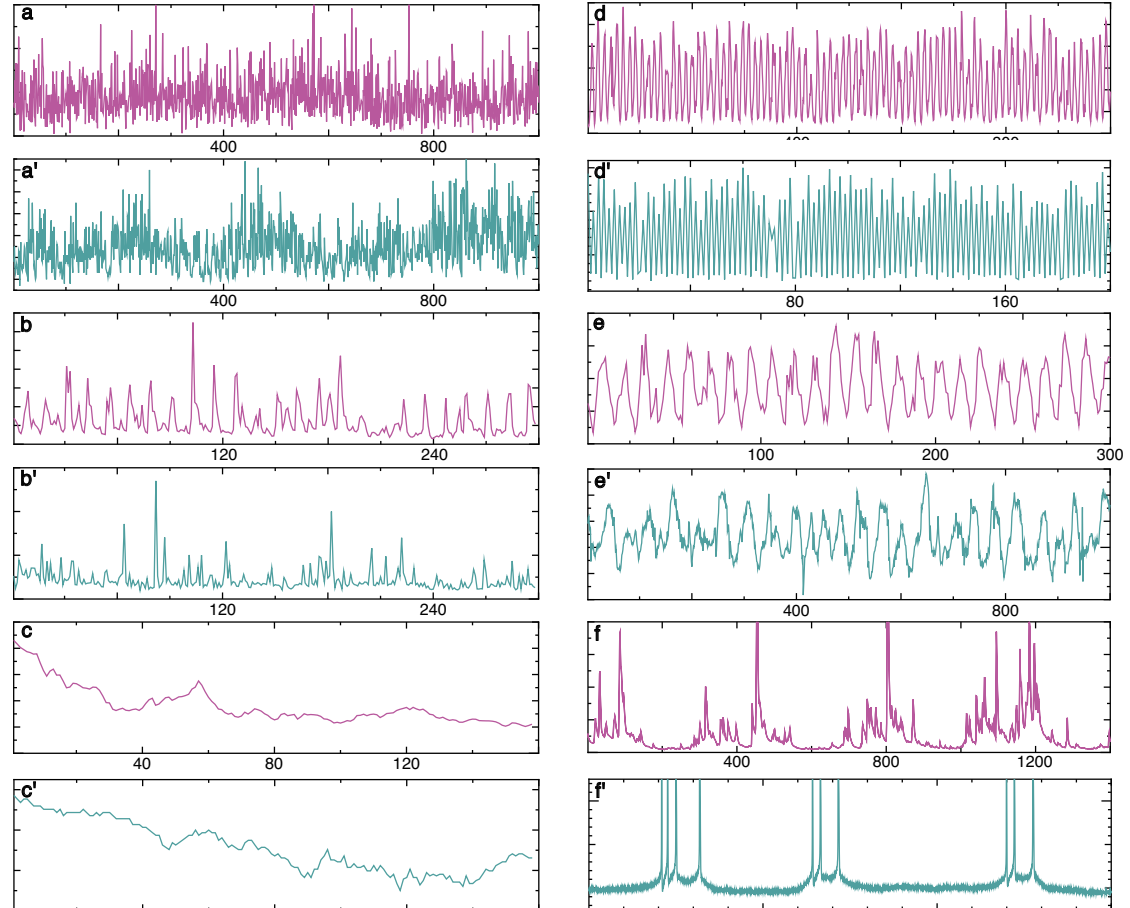

a and a' One for precipitatition (Philadelphia in $1820-01$ to $1903-04$ ) and the other for heartbeats with
ventricular tachycardia (0209.v1). band b' One for peak streamflow (Wolf River at New London, 1914 01 to 1938-10) and the other for brainwaves in a brainstorm project. c and $c^{\prime}$ ' ne for ex exchange rate

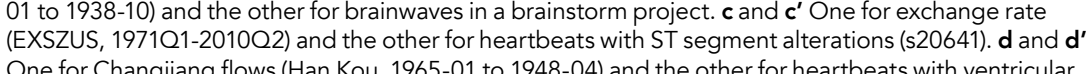
One for Changijiang flows (Han Kou, 1965-01 to $1948-044$ and the other for heartbeats with ventricular
fibililation (0217.vif). e and $e^{\prime}$ One for ozone thickness (Switzerland, 1926-01 to 1952-12) and the other for heartheats fron older adut f(1004). fand $f^{\prime}$ One for Saugeen River flows (Port Elgin, 1988-01-01 for heartbeats from an older adult (f1004). Fand $f^{\prime}$ One tor Saugeee
to $1991-11-01)$ and the other for nerve impulses from a snail neuron range dependence and self-similarity with power law were determined by the comparative strength of external environmental and internal organisationa influences. If the observation scale is larg enough, or the frequency of data is low frough, it is possible to observe typical model reveled thatastoch sic (randon table fixed point had both self-similatity and long-term memory properties, while deterministic (non-random) stable fixed

\section{EFFECTS OF}

\section{NONLINEAR DYNAMICS}

Plotting the data generated by NLARI revealed that the fractal level of a stable fixed point is controlled by the wave strength of externg the rive arger of la whereas a smaller amplitude indicator disclosed a higher level of self-similarity

Non-integer fractal dimensions do not explain what creates fractal behaviour and controls fractal levels.

point usually only showed self-similarity. Both stochastic and deterministic period cycles together with chaos only display long-term memory. The length of the restorative delays also had a significan whect with self-similanty being observed When the restorative delay was an even

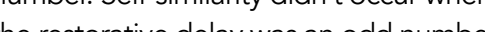

Additionally, a larger amplitude indicator or an even restorative delay could make the sample autocorrelation function oscillate. Professor He observed that the fractal levels of period cycles and chaos relied on the intrinsic resistance, restoration, and regulative delays. Once the cycles or chaos, however, the extrinsic disturbances can be ignored.

Contrary to the underpinning

Constamension,

作 suggest that fractal of self-reand systems can in fact be measured

by integer dimensions.

Professor He also indicates that other similar fractals and dynamics occur in different disciplines and that these could also be characterised by the NLARI process that she has uncovered by applying Newton's second law to self-regulating systems. Professo He's research suggests that the perasiveness of similarity in dynamics, or fractals, of nonlinear models in areas such as modelling rainfall, river stream low, ozone thickness, brainwaves and nerve impulses could indicate that Newton's second law

\section{Behind the Research}

\section{Dr Zonglu He}

E: zongluhe@kaetsu.ac.jp T: $+81424463711 \quad$ W: http://www.kaetsu.ac.jp/
W: https://wwww researchgate net/profile/Zonglu

Research Objectives

Professor He's research focuses on control mechanism of nonlinear dynamics.

\section{Detail}

Faculty of Management and Economics Kaetsu University hakoganei, Kodaira-shi Tokyo $187-8578$

Bio

Dr Zonglu He is a professor of Economics at Kaetsu University. She received her PhD from Hiroshima University. Her current research interests focus on control mechanism of nonlinear dynamics and fractals by modelling the data generating processes of different fields in integrating knowledge and theories.

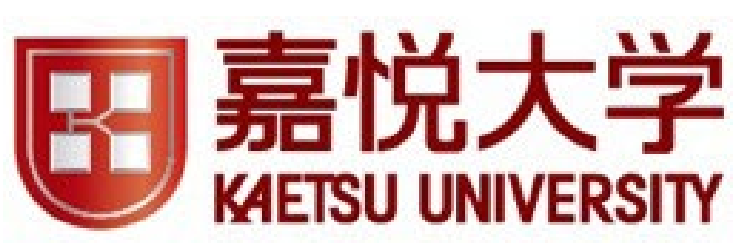

\section{References}

He, Z. L. (2007). A new class of nonlinear integrated models. Far East Journal of Theoretical Statistics, 23, 31-50.

$\mathrm{He}$, Z. L. (2013). Dynamics and stability of a new class of nonlinear integrated models with resilience mechanisms. Far East Jounal of Dynamica Systems, 21 ,

He, Z. L. (2014). Estimation and tests of nonlinear autoregressive integrated models. Far East Journal of Theoretical Statistics, 49, 129-164.

He, Z. L. (2018). Integer-dimensional fractals of nonlinear dynamics, control mechanisms, and physical implications. Scientific Reports, 8, 10.1038/541598-018-286693 3 Ave be [Accessed 7th March 2019]

\section{Personal Response}

\section{What initially prompted your research into integer-}

II Fractal dimension seems not to be a physical scale condition as a physical scale. If fractals model complex physical processes and dynamical systems, the nonlinear dynamics and the control physical laws for these fractaldimension physical processes should result from fractadimension spacetimes. Alternatively, fractals may occur
in integer-dimension spacetimes.

\section{What are your plans for future developments with} III I will use the NLARI model to extract depth information parameter alterations from heartbeat series to assess cardiac, metabolic, and autonomic nervous functions and the risk for cardiovascular diseases; and explore information
transmission in cortical circuits. 